www.mdpi.com/journal/applsci

Article

\title{
Electrical Properties of Graphene for Interconnect Applications
}

\section{Antonio Maffucci ${ }^{1,2, *}$ and Giovanni Miano ${ }^{3}$}

1 Department of Electrical and Information Engineering, University of Cassino and Southern Lazio, via G. Di Biasio 43, Cassino 03043, Italy

2 INFN-LNF, Frascati National Laboratory, National Institute of Nuclear Physics, via E. Fermi 40, Frascati 00044, Italy

3 Department of Electrical Engineering and Information Technology, University Federico II of Naples, via Claudio 21, Napoli 80125, Italy; E-Mail: miano@unina.it

* Author to whom correspondence should be addressed; E-Mail: maffucci@uniclam.it; Tel.: +39-0776-299-3691; Fax: +39-0776-299-3707.

Received: 23 March 2014; in revised form: 14 May 2014 / Accepted: 14 May 2014 /

Published: 30 May 2014

\begin{abstract}
A semi-classical electrodynamical model is derived to describe the electrical transport along graphene, based on the modified Boltzmann transport equation. The model is derived in the typical operating conditions predicted for future integrated circuits nano-interconnects, i.e., a low bias condition and an operating frequency up to $1 \mathrm{THz}$. A generalized non-local dispersive Ohm's law is derived, which can be regarded as the constitutive equation for the material. The behavior of the electrical conductivity is studied with reference to a 2D case (the infinite graphene layer) and a 1D case (the graphene nanoribbons). The modulation effects of the nanoribbons' size and chirality are highlighted, as well as the spatial dispersion introduced in the $2 \mathrm{D}$ case by the dyadic nature of the conductivity.
\end{abstract}

Keywords: graphene; graphene nanoribbons; nano-interconnects

\section{Introduction}

Due to their outstanding physical properties, graphene and its allotropes (carbon nanotubes (CNTs) and graphene nanoribbons (GNRs)) are the major candidates to become the silicon of the 21 st century and open the era of so-called carbon electronics [1,2]. 
Amongst all such applications, the low electrical resistivity, high thermal conductivity, high current carrying capability, besides other excellent mechanical properties, make graphene a serious candidate to replace conventional materials in realizing VLSI nano-interconnects [3,4]. A carbon-based VLSI technology is not yet a pure theoretical wish, since in the last few years, the rapid progress in graphene fabrication made possible the first examples of real-world applications. The works [5,6] present high frequency CMOS oscillators integrating CNT or GNR interconnects, whereas [7] shows the first example of a computer with PMOS transistors entirely made of CNTs. Carbon nanotubes or graphene interconnects are also successfully integrated into innovative organic transistors for flexible plastic electronics [8].

The increasing interest in carbon nano-interconnects leads to the quest for more and more accurate and reliable models, able to include all the quantum effects arising at the nanoscale. This topic has been given great attention by the recent literature, which presented several modeling approaches, like phenomenological [9] and semi-classical ones [10]. Based on such models, many papers predicted that carbon materials could outperform copper for IC on-chip interconnects and vias [11-16].

In the typical working conditions of nano-interconnects, namely a frequency up to $\mathrm{THz}$ and low bias conditions, such nano-structures do not exhibit tunneling transport. Thus, the electrodynamics may be studied by using a semi-classical description of the electron transport. This leads to the derivation of a constitutive relation between the electrical field and the density of the current in the form of a generalization of the classical Ohm's law, introducing non-local interactions and dispersion. By coupling such a relation to Maxwell equations, it is possible to derive a generalized transmission line model for such nano-interconnects. This approach has been followed by the authors to model isolated metallic CNTs in [16], CNTs with arbitrary radius and chirality in [17,18], multi-walled CNTs [19] and GNRs [20]. The semi-classical model gives results consistent with those provided by an alternative hydrodynamic model [21].

In this paper, a comprehensive and self-consistent analysis of the electrical properties of graphene is presented, with special emphasis on its application as an innovative material for nano-interconnects. The self-consistency is guaranteed by the fact that the used transport model is derived from an electrodynamical model described in terms of physically meaningful parameters. This differs from what can be obtained by means of heuristics approaches. In particular, a self-consistent model provides a clear analytical relation between the model parameters and the physical and geometrical properties of the investigated structure. The considered frequency range of applications is limited to values up to $1 \mathrm{THz}$, and the operational conditions fall into the low bias regime (the longitudinal electric field along the interconnect should be $E_{\mathrm{z}}<0.54 \mathrm{~V} / \mu \mathrm{m}$ ). For such cases, interband transitions are absent and non-linear effects are negligible, and thus, a simple linear transport of the conducting electrons ( $\pi$-electrons) may be derived based on the tight-binding model and the semi-classical Boltzmann equation.

Section 2 is devoted to a brief résumé of the main properties of the band structure for a graphene sheet and for a graphene nanoribbon. In Section 3, the transport model is presented, derived from a semi-classical model based on the linearized Boltzmann transport equation. The model leads to the constitutive relation for the graphene, written in terms of a generalized non-local Ohm's law in the frequency and wave-number domain. Section 4 presents a detailed discussion about the properties of the electrical conductivity, which, in general, results in being a dyad. 


\section{Band Structure}

In this chapter, we briefly summarize the main features of the band structure, first for an infinite graphene layer (a 2D structure) and, then, for the graphene nanoribbon (a 1D structure).

\subsection{Graphene Layer}

Figure 1a shows the Bravais lattice of the graphene. The unit cell, $S_{\mathrm{g}}$, is spanned by the two vectors, $\mathbf{a}_{1}$ and $\mathbf{a}_{2}$, and contains two carbon atoms. The basis vectors $\left(\mathbf{a}_{1}, \mathbf{a}_{2}\right)$ have the same length, $\left|\mathbf{a}_{1}\right|=\left|\mathbf{a}_{2}\right|=a=\sqrt{3} b$, and form an angle of $\pi / 3$, where $b=1.42 \AA$ is the interatomic distance. The components of the vectors, $\mathbf{a}_{1}$ and $\mathbf{a}_{2}$, with respect to the rectangular coordinate system, $(0, x, y)$ are, respectively, $\left(\sqrt{3} a_{0} / 2, a_{0} / 2\right)$ and $\left(\sqrt{3} a_{0} / 2,-a_{0} / 2\right)$. The area of the unit cell, $S_{\mathrm{g}}$, is $A_{g}=\sqrt{3} a_{0}^{2} / 2$.

Figure 1. The structure of graphene. (a) Bravais lattice; (b) reciprocal lattice.

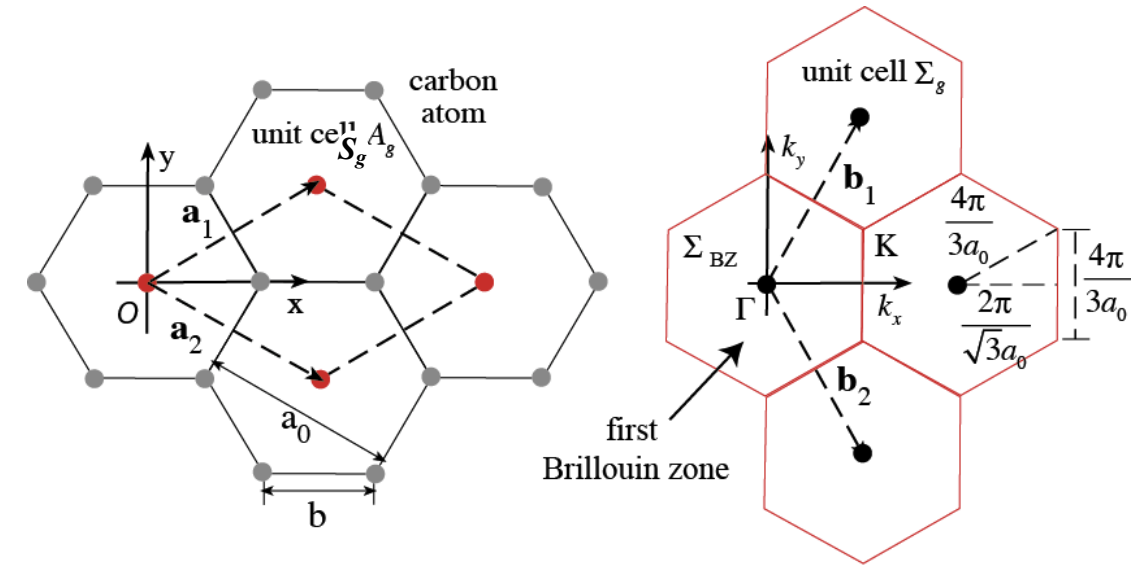

(a) Bravais lattice

(b) Reciprocal lattice

In the reciprocal $k$-space depicted in Figure $1 b$, the graphene is characterized by the unit cell, $\sum_{g}$, spanned by the two vectors, $\mathbf{b}_{1}$ and $\mathbf{b}_{2}$, which have the same length $\left|\mathbf{b}_{1}\right|=\left|\mathbf{b}_{2}\right|=b_{0}=4 \pi \sqrt{3} a_{0}$ and form an angle of $2 \pi / 3$. The components of the vectors, $\mathbf{b}_{1}$ and $\mathbf{b}_{2}$, with respect to the rectangular coordinate system $\left(\mathrm{Q}, K_{x}, k_{y}\right)$ are, respectively, $\left(2 \pi / \sqrt{3} a_{0}, 2 \pi / a_{0}\right)$ and $\left(2 \pi / \sqrt{3} a_{0},-2 \pi / a_{0}\right)$. The area of the unit cell, $\sum_{g}$, is $B_{g}=8 \pi^{2} \sqrt{3} \cdot a_{0}^{2}$. The basis vectors of the direct space $\left(\mathbf{a}_{1}, \mathbf{a}_{2}\right)$ and the basis vectors of the reciprocal space $\left(\mathbf{b}_{1}, \mathbf{b}_{2}\right)$ are related by $\mathbf{a}_{i} \cdot \mathbf{b}_{j}=2 \pi \delta_{i j}$ with $i, j=1,2$ and the areas, $A_{\mathrm{g}}$ and $B_{\mathrm{g}}$, are related by $A_{\mathrm{g}} B_{\mathrm{g}}=(2 \pi)^{2}$.

The graphene possesses four valence electrons for each carbon atom. Three of these (the so-called $\sigma$-electrons) form tight bonds with the neighboring atoms in the plane and do not play a part in the conduction phenomenon. The fourth electron (the so-called $\pi$-electron), instead, may move freely between the positive ions of the lattice.

In the nearest-neighbors tight-binding approximation, the $\pi$-electrons energy dispersion relation is

$$
E^{( \pm)}(\mathbf{k}) \pm \gamma\left[1+4 \cos \left(\frac{\sqrt{3} k_{x} a_{0}}{2}\right) \cos \left(\frac{k_{y} a_{0}}{2}\right)+4 \cos ^{2}\left(\frac{k_{y} a_{0}}{2}\right)\right]^{1 / 2}
$$


where $E^{( \pm)}$denotes the energy, the + sign denotes the conduction band, the - sign denotes the valence band and $\gamma=2.7 \mathrm{eV}$ is the carbon-carbon interaction energy. The electronic band structure is depicted in Figure 2: the valence and conduction bands touch each other at the six vertices of each unit cell, the so-called Fermi points. In the neighborhood of each Fermi point, the energy dispersion relation may be approximated as:

$$
E^{( \pm)} \approx \pm \hbar v_{F}\left|\mathbf{k}-\mathbf{k}_{0}\right|
$$

where $\mathbf{k}_{0}$ is the wavenumber at a Fermi point, $v_{F} \approx 0.87 \times 10^{6} \mathrm{~m} / \mathrm{s}$ is the Fermi velocity of the $\pi$-electrons and $\hbar$ is the Planck constant.

Figure 2. Graphene electronic band structure. Inset: the neighborhood of a Fermi point.

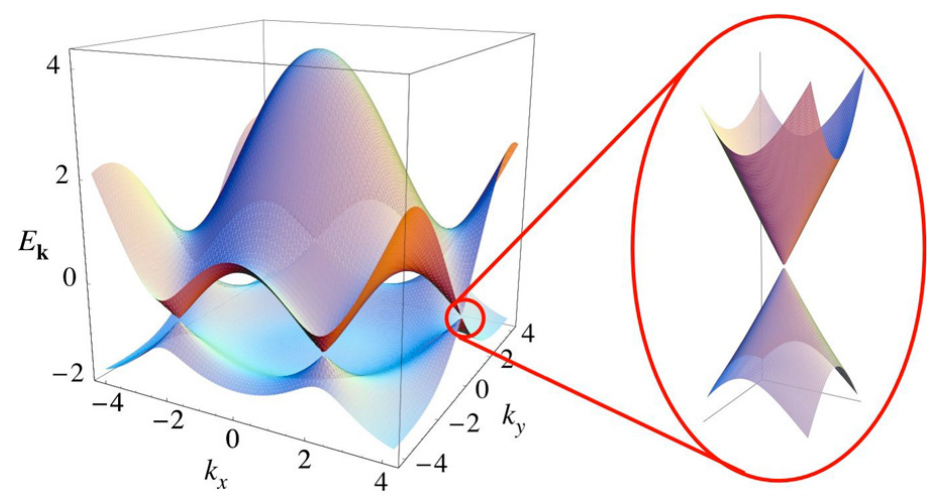

In the ground state, the valence band of the graphene is completely filled by the $\pi$-electrons. In general, at equilibrium, the energy distribution function of $\pi$-electrons is given by the Dirac-Fermi function:

$$
F\left[E^{( \pm)}\right]=\frac{1}{e^{E^{( \pm)} / k_{B} T_{0}}+1}
$$

where $k_{\mathrm{B}}$ is the Boltzmann constant and $T_{0}$ is the graphene absolute temperature, the electrochemical potential of the graphene being null valued. The distribution function differs from the ideal distribution function $F\left[E^{( \pm)}\right]=u\left[-E^{( \pm)}\right]$, where $u=u(x)$ is the Heaviside function, only in a region of order $k_{\mathrm{B}} T_{0}$ around the point $E^{( \pm)}=0$; at room temperature, it results $k_{B} T_{0} \cong 0.02 \mathrm{eV}$.

\subsection{Graphene Nanoribbons}

Let us now discuss the so-called graphene nanoribbons (GNRs), i.e., ribbons obtained by cutting a graphene layer, characterized by a high aspect-ratio, namely a transverse width, $w$, much smaller than the longitudinal ribbon length. Figure 3 shows the two basic shapes for GNRs, namely nanoribbons with armchair edges and nanoribbons with zigzag edges (e.g., [20]). These edges have a $30^{\circ}$ difference in their orientation within the graphene sheet. We assume that all dangling bonds at graphene edges are terminated by hydrogen atoms and, thus, do not contribute to the electronic states near the Fermi level. In the following, we will assume the Fermi energy to be zero. However, this level may move to values such as $0.2-0.4 \mathrm{eV}$, considering the interactions at the GNR/substrate interface. 
Figure 3. The structure of graphene nanoribbons. (a) Armchair edges; (b) zigzag edges.

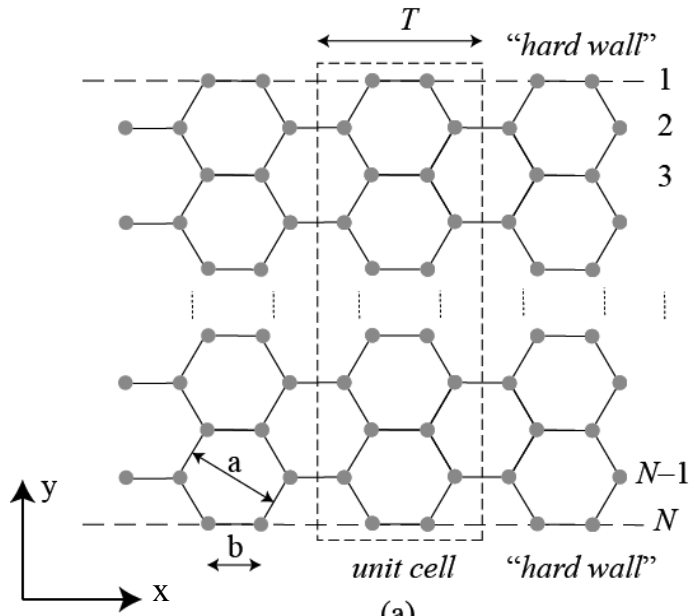

(a)

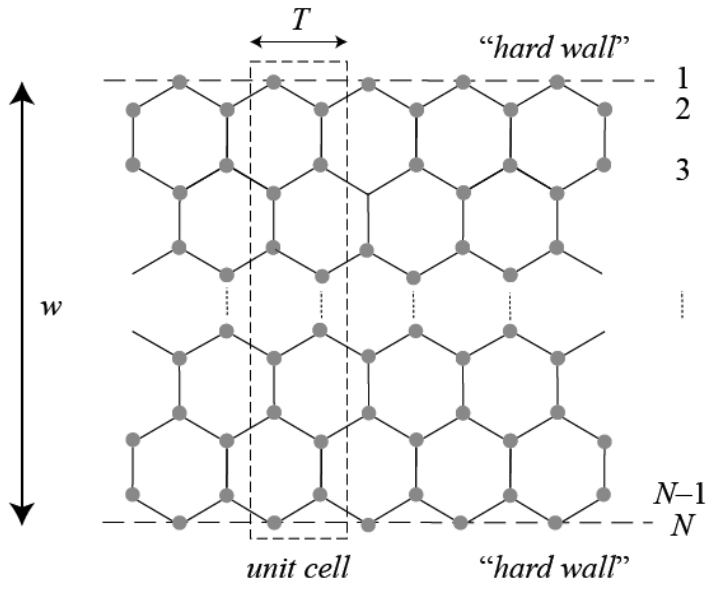

(b)

As shown in Figure 3, the width, $w$, of a graphene nanoribbon is directly related to the integer, $N$, indicating the number of dimers (two carbon sites) for the armchair nanoribbons and the number of zigzag lines for the zigzag nanoribbons. For armchair GNRs, the unit cell has a length $T=\sqrt{3} a$ and a width $w=N a / 2$, whereas for zigzag ones, it is $T=a$ and $w=\sqrt{3} N a / 2$.

Given the number $N$ and, thus, the GNR width, $w$, the energy spectrum of the $\pi$-electrons can be obtained by slicing the band structure of graphene in Figure 2 (e.g., [20,22,23]). Figure 4 shows the band structure for an armchair GNR, assuming $N=5$ and $N=6$. For $N=3 q-1(q=1,2, \ldots)$, there exists a sub-band, $\mu$, for which the direct bandgap is zero; hence, the GNR behaves as a metal. For other values, the armchair GNRs is semiconducting. However, the direct bandgap decreases with $w$ increasing and approaches zero in the limit of very large $w$, consistent with the behavior of the graphene layer reported in Section 2.1. Therefore, the value of the width (hence, the number, $N$ ) determines whether the nanoribbon is metallic or semiconducting.

Figure 4. Energy band structure of an armchair nanoribbon versus the normalized wavenumber $k^{\prime}=k l / \pi$. (a) $N=5$; (b) $N=6$.
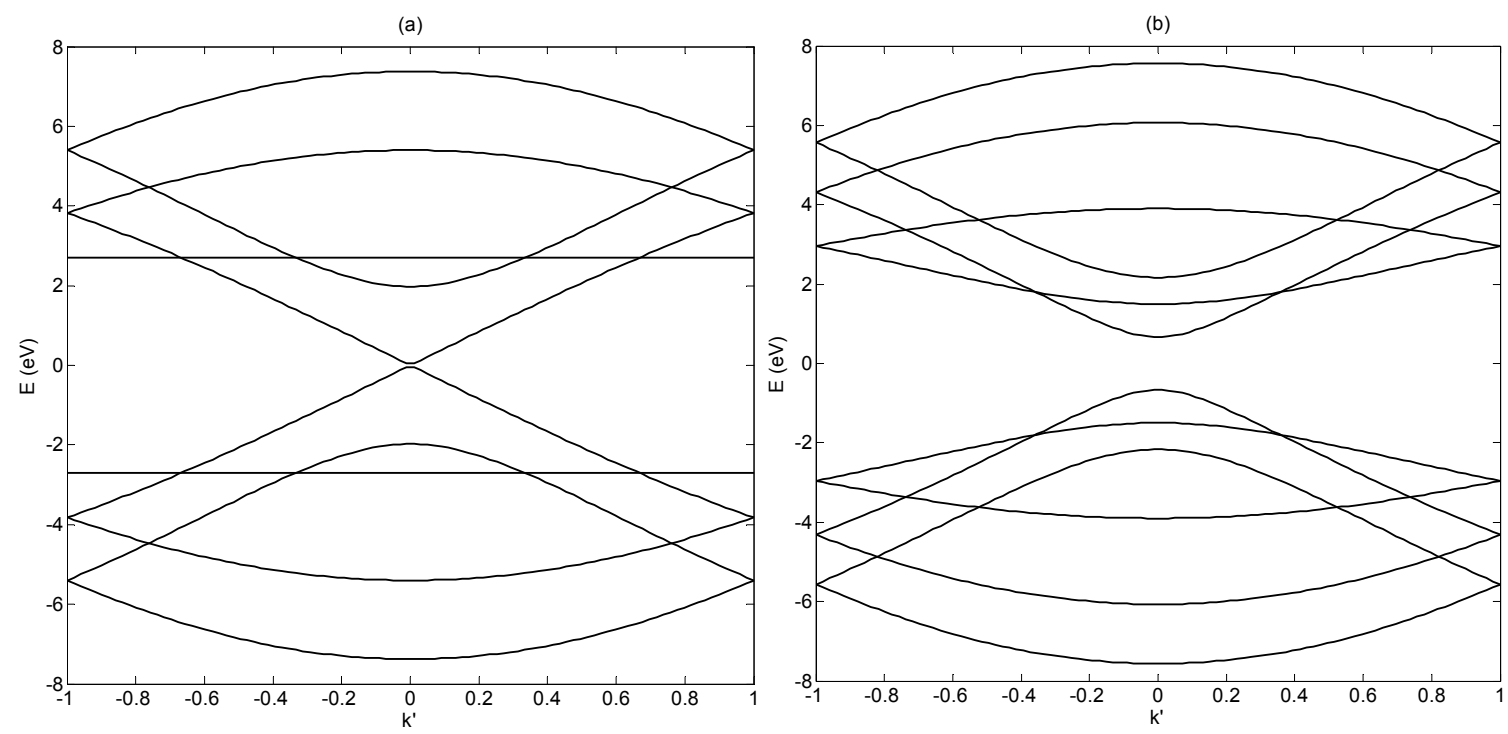
Figure 5 refers to a zigzag GNR, assuming $N=5$ and $N=20$ : such nanoribbons have partially flat bands owing to the edge states; hence, their bandgap is always zero, and their behavior is always metallic.

Figure 5. The energy band structure of a zigzag nanoribbon versus the normalized wavenumber $k^{\prime}=k l / \pi$. (a) $N=5$; (b) $N=20$.
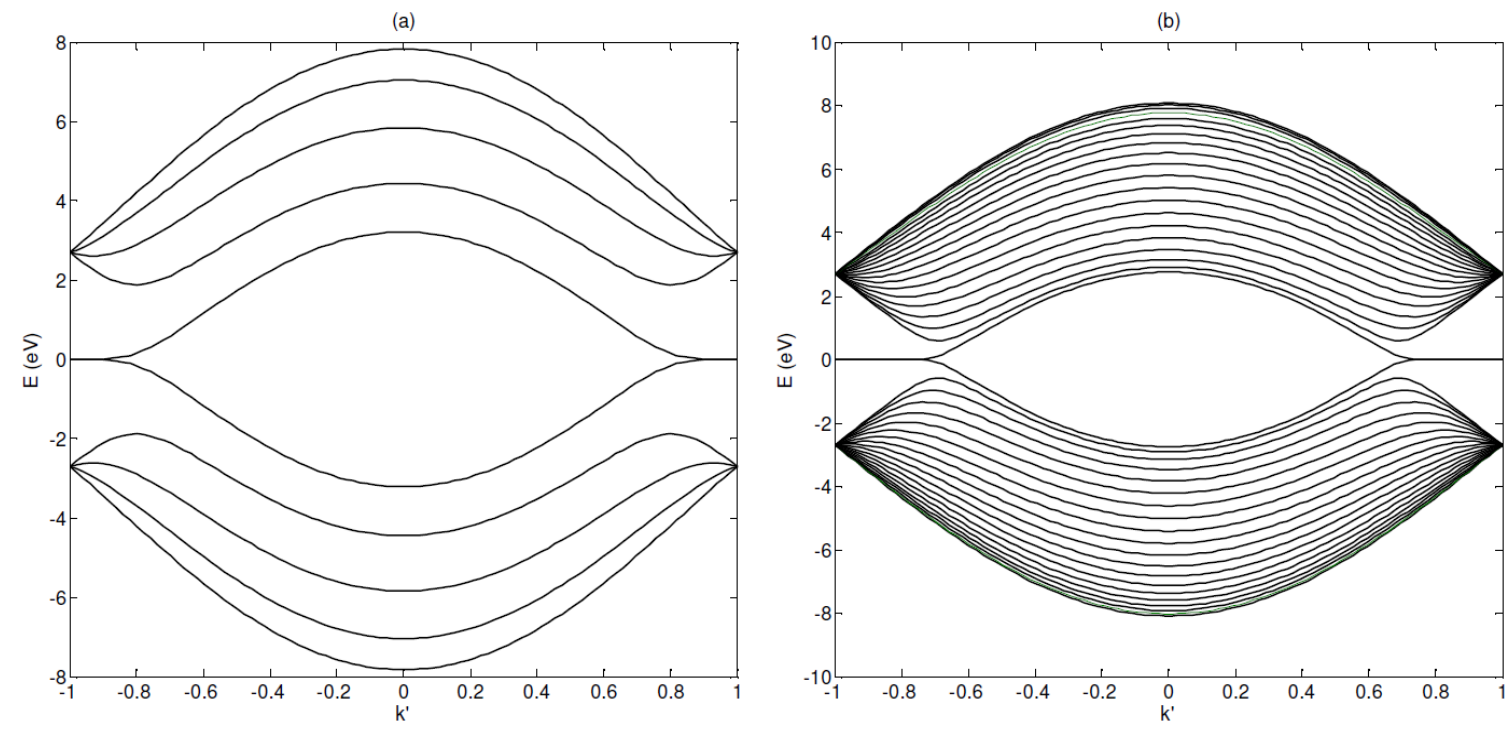

\section{Transport Model}

We model the propagation of an electromagnetic wave along a graphene nanoribbon in the low frequency regime, where only intraband transitions of $\pi$-electrons with unchanged transverse quasi momentum are allowed. These transitions contribute to the axial conductivity, but not to the transverse conductivity. For typical dimensions of the nano-interconnects for the $14 \mathrm{~nm}$ technology node and below, this assumption limits the model to a frequency up to some THz. Following the stream of what is done in $[18,19,24]$, in such a condition, the electrodynamics of the $\pi$-electrons may be described by the quasi-classical Boltzmann equation. In the following, we start from the 2D case (graphene infinite layer) and then particularize the results to the 1D case (GNRs).

\subsection{Transport Model for the Graphene Layer}

The $\pi$-electrons in an infinite graphene layer behave as a two-dimensional electron gas moving on the surface under the action of the electric field. We disregard the Lorentz force term due to the magnetic field, because it does not contribute to the linear response of the electron gas.

The electron gases are described by the distribution functions $f^{( \pm)}=f^{( \pm)}(\mathbf{r}, \mathbf{k}, t)$, where $\mathbf{r}$ is the vector position in the direct space with the origin at $O, \mathbf{k}$ is the electron wave-vector restricted to the first Brillouin zone and $\sum_{\mathrm{BZ}}$ and $t$ represents the time variable. Here, the + sign denotes the conduction band, the - sign denotes the valence band.

Each of these distribution functions satisfies the quasi-classic Boltzmann kinetic equation, 


$$
\frac{\partial f^{( \pm)}}{\partial t}+\mathbf{v}^{( \pm)} \cdot \frac{\partial f^{( \pm)}}{\partial \mathbf{r}}+\frac{e}{\hbar} \mathbf{E}_{t} \cdot \frac{\partial f^{( \pm)}}{\partial \mathbf{k}}=-v\left(f^{( \pm)}-f_{0}^{( \pm)}\right)
$$

where $e$ is the electron charge, $\mathbf{E}_{t}=\mathbf{E}_{t}(\mathbf{r}, t)$ represents the tangential component of the electric field at the graphene surface, $v$ is the relaxation frequency $\left(v \cong 5 \cdot 10^{11} s^{-1}\right)$ and $\mathbf{v}^{( \pm)}=\mathbf{v}^{( \pm)}(\mathbf{k})$ is the velocity of the $\pi$-electrons in the conduction/valence band given by:

$$
\mathbf{v}^{( \pm)}(\mathbf{k})=\frac{1}{\hbar} \frac{\partial E^{( \pm)}}{\partial \mathbf{k}}
$$

The symbols, $\partial / \partial \mathbf{r}$ and $\partial / \partial \mathbf{k}$, denote the gradients with respect to the variables, $\mathbf{r}$ and $\mathbf{k}$, respectively. The distribution functions of the $\pi$-electrons at equilibrium are:

$$
f_{0}^{( \pm)}(\mathbf{k})=\frac{1}{2 \pi^{2}} F\left[E^{( \pm)}(\mathbf{k})\right]
$$

Let us consider a time-harmonic field, in the form $\mathbf{E}_{t}(\mathbf{r}, t)=\operatorname{Re}\left\{\hat{\mathbf{E}}_{t} \exp [i(\omega t-\boldsymbol{\beta} \cdot \mathbf{r})]\right\}$, where $\beta$ is the wave-vector defined in the two-dimensional space. Let us set $f^{( \pm)}=f_{0}^{( \pm)}(\mathbf{k})+\delta f^{( \pm)}(\mathbf{r}, \mathbf{k}, t)$ and consider a time-harmonic perturbation $\delta f^{( \pm)}(\mathbf{r}, \mathbf{k}, t)=\operatorname{Re}\left\{\delta f_{1}^{( \pm)}(\mathbf{k} ; \omega, \boldsymbol{\beta}) \exp [i(\omega t-\boldsymbol{\beta} \cdot \mathbf{r})]\right\}$, where $\delta f_{1}^{( \pm)}$is a small quantity to be found: using Equation (4) and retaining only the first order terms, we obtain:

$$
\delta f_{1}^{( \pm)}=i \frac{e}{\hbar} \frac{1}{\left(\omega-\mathbf{v}^{( \pm)} \cdot \beta-i v\right)} \hat{\mathbf{E}}_{t} \cdot \frac{\partial f_{0}^{( \pm)}}{\partial \mathbf{k}}
$$

Let us now introduce the time harmonic surface current density $\mathbf{j}(\mathbf{r}, t)=\operatorname{Re}\{\hat{\mathbf{J}}(\omega, \boldsymbol{\beta}) \exp [i(\omega t-\boldsymbol{\beta} \cdot \mathbf{r})]\}$ : its amplitude, $\hat{\mathbf{J}}(\omega, \boldsymbol{\beta})$, can be computed by summing the contributions from all the sub-bands in the first Brillouin zone:

$$
\hat{\mathbf{J}}(\omega, \boldsymbol{\beta})=\int_{\Sigma_{B Z}}\left[e \mathbf{v}^{(-)}(\mathbf{k}) \delta f_{1}^{(-)}(\mathbf{k} ; \omega, \boldsymbol{\beta})+e \mathbf{v}^{(+)}(\mathbf{k}) \delta f_{1}^{(+)}(\mathbf{k} ; \omega, \boldsymbol{\beta})\right] d^{2} \mathbf{k}
$$

Here, the first term is related to the valence bands and the second one to the conduction bands. By combining Equations (7) and (8) we get the constitutive relation of the medium:

$$
\hat{\mathbf{J}}=\vec{\sigma}(\omega, \beta) \hat{\mathbf{E}}_{t}
$$

This can be regarded as a generalized Ohm's law, for which the conductivity is a symmetric dyad, given by:

$$
\ddot{\boldsymbol{\sigma}}(\omega, \boldsymbol{\beta})=\frac{i e^{2}}{\hbar} \int_{\Sigma_{B z}}\left[\frac{\mathbf{v}^{(-)}(\mathbf{k})}{\left[\omega-\mathbf{v}^{(-)}(\mathbf{k}) \cdot \boldsymbol{\beta}-i v\right]} \frac{\partial f_{0}^{(-)}}{\partial \mathbf{k}}+\frac{\mathbf{v}^{(+)}(\mathbf{k})}{\left[\omega-\mathbf{v}^{(+)}(\mathbf{k}) \cdot \boldsymbol{\beta}-i v\right]} \frac{\partial f_{0}^{(+)}}{\partial \mathbf{k}}\right] d^{2} \mathbf{k}
$$

The function:

$$
\Delta(E)=\frac{d F}{d E}=-\frac{1}{4 k_{B} T_{0}} \frac{1}{\cosh ^{2}\left(E / 2 k_{B} T_{0}\right)}
$$

is an even function. As a consequence of this property and of the right-left symmetry of the energy dispersion curves with respect to the point $\mathbf{k}=0$, the sum in the r.h.s. of Equation (10) is twice the contribution of the conduction bands, hence:

$$
\tilde{\sigma}(\omega, \boldsymbol{\beta})=i \frac{e^{2}}{\pi^{2}} \int_{\Sigma_{B z}} \frac{\mathbf{v}(\mathbf{k}) \mathbf{v}(\mathbf{k})}{\omega-\mathbf{v}(\mathbf{k}) \cdot \boldsymbol{\beta}-i V} \Delta[\mathcal{E}(\mathbf{k})] d^{2} \mathbf{k}
$$


Note that the function, $\Delta$, behaves as an impulsive function centered at $E=0$; hence, only the neighbors of the Fermi points with radius $k_{\text {eff }}=4 k_{B} T_{0} / \hbar v_{F}$ and belonging to the first Brillouin zone give a meaningful contribution: let us indicate with $C_{F}$ the circumference of radius $k_{\text {eff }}$ centered at the Fermi point with electron wave vector $\mathbf{K}$. In addition, assuming $\mathbf{v}\left(\mathbf{k}^{\prime}\right) \cong v_{F} \hat{\mathbf{k}}^{\prime}$, where $\mathbf{k}^{\prime} \equiv \mathbf{k}-\mathbf{K}$, we get the final expression:

$$
\ddot{\sigma}(\omega, \boldsymbol{\beta})=i \frac{2 e^{2}}{\pi^{2}} v_{F}^{2} \int_{C_{F}} \frac{\hat{\mathbf{k}}^{\prime} \hat{\mathbf{k}}^{\prime}}{\omega-v_{F} \hat{\mathbf{k}}^{\prime} \cdot \boldsymbol{\beta}-i V} \Delta\left[\mathcal{E}\left(\mathbf{k}^{\prime}\right)\right] d^{2} \mathbf{k}^{\prime}
$$

\subsection{Transport Model for a Graphene Nanoribbon}

Along a graphene nanoribbon, in the operating conditions assumed here, the $\pi$-electrons mainly move along the longitudinal axis, i.e., the transverse components may be neglected. Therefore, the transport model is derived from that exposed in Section 3.1, assuming a 1D case: the electrons are quantum confined laterally and, thus, occupy quantized energy levels, whereas along the longitudinal axis, the wave-vector, $k$, is assumed to be almost continuous.

If $\mathrm{T}$ is the translational length of the unit cell (see Figure 3), the first Brillouin zone is given by the interval $(-\pi / T, \pi / T)$ and the longitudinal conductivity in the spatial and wavenumber domain obtained from Equation (13) is given by the sum of the contribution of the $N$ conduction sub-bands in the circumference $C_{F}$ :

$$
\hat{\sigma}_{x x}(\beta, \omega)=\sum_{\mu=1}^{N} \hat{\sigma}_{\mu}(\beta, \omega)
$$

where:

$$
\hat{\sigma}_{\mu}(\beta, \omega)=i \frac{4 e^{2}}{\pi W} \int_{0}^{-\pi / T} \frac{v_{\mu}^{(+)^{2}}(k)}{1-v_{\mu}^{(+)^{2}}(k) \frac{\beta^{2}}{\left(\omega-i v_{\mu}\right)^{2}}} \frac{d F}{d E_{\mu}^{(+)}} d k
$$

Using the above results, the generalized Ohm's law Equation (9) may be expressed for a GNR as follows:

$$
\left[1-\psi(\omega) \beta^{2}\right] \hat{J}_{z}(\beta, \omega)=\frac{\sigma_{0}}{1+i \omega / \nu} \hat{E}_{x}(\beta, \omega)
$$

where we have introduced the following functions:

$$
\psi(\omega)=\frac{\alpha(\omega) v_{F}^{2}}{v^{2}(1+i \omega / v)^{2}}, \quad \sigma_{0}=\frac{2 v_{F} M}{v R_{0} \pi W}
$$

$R_{0}=12.9 \mathrm{k} \Omega$ being the quantum resistance, and:

$$
\begin{gathered}
M=\frac{2 \hbar}{v_{F}} \sum_{\mu=1}^{N} \int_{0}^{\pi / l} v_{\mu}^{(+) 2}(k)\left(-\frac{d F}{d E_{\mu}^{(+)}}\right) d k, \\
\alpha(\omega)=\frac{2}{M} \frac{\hbar}{v_{F}^{3}} \sum_{\mu=0}^{n-1} \int_{0}^{\pi / l} v_{\mu}^{(+) 4}(k)\left(-\frac{d F}{d E_{\mu}^{(+)}}\right) d k
\end{gathered}
$$


The quantity $M$ in Equation (19) is the equivalent number of conducting channels, a measure of the number of sub-bands that effectively contribute to the electric conduction, i.e., those that cross or are closer to the Fermi level. A detailed discussion on the behavior of such a number $M$ for CNTs and GNRs may be found in [18-20], where it is clearly shown how such a quantity strongly depends on the chirality, size and temperature of such carbon nanostructures.

\section{Discussion}

\subsection{Electrical Conductivity in the Long Wavelength Limit}

Let us first assume the case of a graphene layer in the long wavelength limit $\beta=0$, for which Equation (13) reduces to:

$$
\ddot{\boldsymbol{\sigma}}(\omega, \mathbf{0})=i \frac{2 e^{2}}{\pi^{2}} v_{F}^{2} \frac{1}{\omega-i V} \int_{C_{F}} \hat{\mathbf{k}}^{\prime} \hat{\mathbf{k}}^{\prime} \Delta\left[\mathcal{E}\left(\mathbf{k}^{\prime}\right)\right] d^{2} \mathbf{k}^{\prime}
$$

In the reference system indicated in Figure 1, the four components, $\sigma_{x x}(\omega, 0), \sigma_{y y}(\omega, 0), \sigma_{x y}(\omega, 0)$ and $\sigma_{y x}(\omega, 0)$ of the dyad, $\sigma(\omega, 0)$, reduce to the following expressions:

$$
\sigma_{x x}(\omega, 0)=\sigma_{y y}(\omega, 0)=\sigma(\omega), \sigma_{x y}(\omega, 0)=\sigma_{y x}(\omega, 0)=0
$$

where:

$$
\sigma(\omega)=\frac{\sigma_{c}}{1+i \omega / v}
$$

In the same condition, the generalized Ohm's law for a graphene nanoribbon Equation (16) would reduce to:

$$
\hat{J}_{x}(\beta, \omega)=\frac{\sigma_{0}}{1+i \omega / \nu} \hat{E}_{x}(\beta, \omega)
$$

Figure 6. Equivalent number of conducting channels for metallic and semiconducting armchair graphene nanoribbons (GNRs) at $300 \mathrm{~K}$.

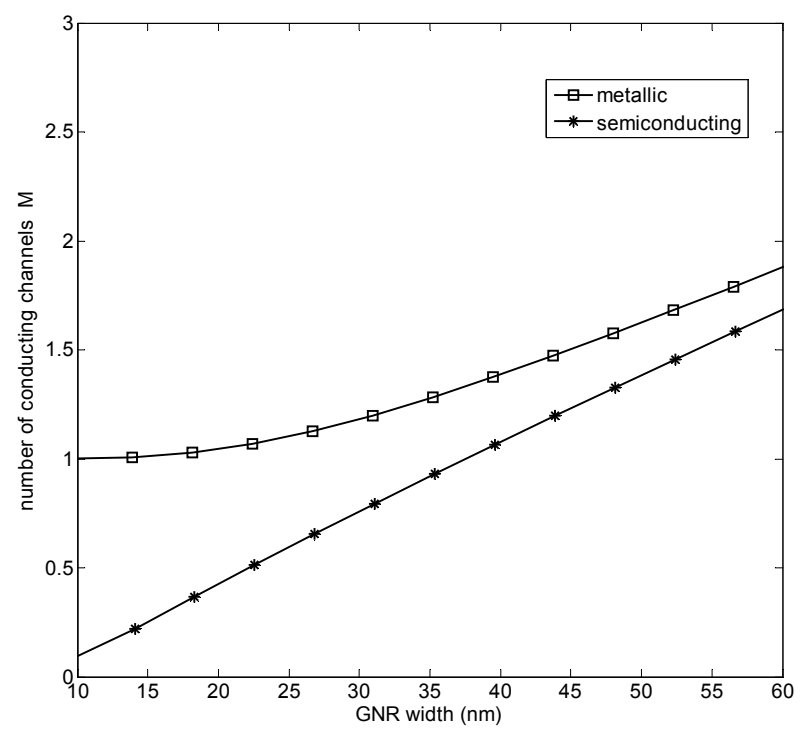


The conductivity, $\sigma_{c}$, in Expression (22) is consistent with $\sigma_{0}$ in Equation (23): $\sigma_{c}$ may be obtained from $\sigma_{0}$ by taking the limit $W \rightarrow \infty$, i.e., when the transverse size of the GNR tends to infinity. To explain this behavior, Figure 6 shows the computed value of the number of conducting channels, $M$, in Equation (18), for a metallic and semiconducting armchair GNR vs. the GNR width. For smaller values of $W$, the contribution to the conductivity given from the semiconducting GNRs is negligible, but it becomes non-negligible as $W$ increases. Figure 7, instead, shows the behavior of the ratio, $\sigma_{0} / \sigma_{c}$, as a function of $W$, which proves the consistency stated above.

Figure 7. The ratio, $\sigma_{0} / \sigma_{c}$, for metallic and semiconducting armchair GNRs at $300 \mathrm{~K}$.

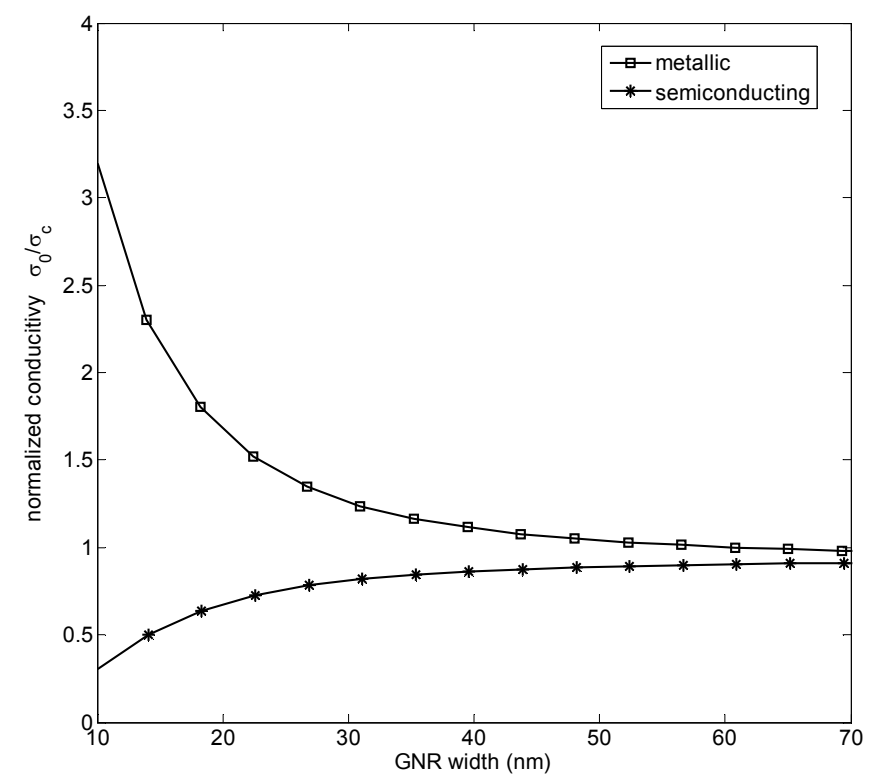

As a conclusion, in the case of a long wavelength limit, the conductivity of graphene does not show spatial dispersion, but only frequency dispersion, according to Equation (22). For a GNR (as in Figure 3), the conductivity may be assumed to have only the longitudinal component $\sigma_{x x}(\omega, 0)=\sigma(\omega)$.

\subsection{Electrical Conductivity in the General Case: Spatial Dispersion}

In the general case, we must take into account the dyadic nature of the conductivity. For the sake of simplicity, let us refer to the components of any vector to the parallel and orthogonal directions with respect to the vector, $\boldsymbol{\beta}$, denoting them with the symbols " || " and " $\perp$ ", respectively. In this system, the four components, $\sigma_{|| \mid}(\omega, \boldsymbol{\beta}), \sigma_{\perp \perp}(\omega, \boldsymbol{\beta}), \sigma_{\| \perp}(\omega, \boldsymbol{\beta})$ and $\sigma_{\perp||}(\omega, \boldsymbol{\beta})$, of the dyad, $\sigma(\omega, \boldsymbol{\beta})$, reduce to the following expressions:

$$
\begin{gathered}
\sigma_{||||}(\omega, \boldsymbol{\beta})=\sigma(\omega) \frac{2}{1+\gamma^{2}+\sqrt{1-\gamma^{2}}}, \quad \sigma_{\perp \perp}(\omega, \boldsymbol{\beta})=\sigma(\omega) \frac{2}{1+\sqrt{1-\gamma^{2}}} \\
\sigma_{\| \perp}(\omega, \boldsymbol{\beta})=\sigma_{\perp||}(\omega, \boldsymbol{\beta})=0,
\end{gathered}
$$

where:

$$
\gamma=\frac{v_{F} \beta}{\omega} \frac{1}{1-v i / \omega}
$$


From such components, it is possible to derive the $x-y$ components, by the simple transformation:

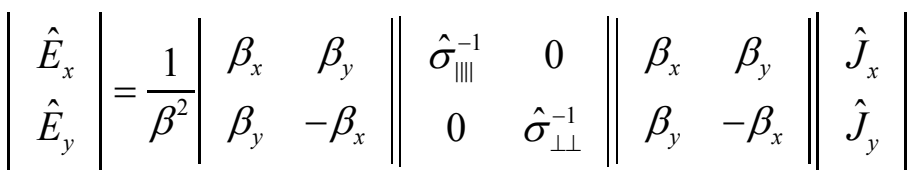

Figure 8. Real (a) and imaginary (b) parts of $\sigma_{\|||}(\omega, \boldsymbol{\beta})$ and $\sigma_{\perp \perp}(\omega, \boldsymbol{\beta})$, normalized to $\sigma_{c}$, $v s .\left(v_{F} / \omega\right) \beta$ for $v / \omega=0.1$.
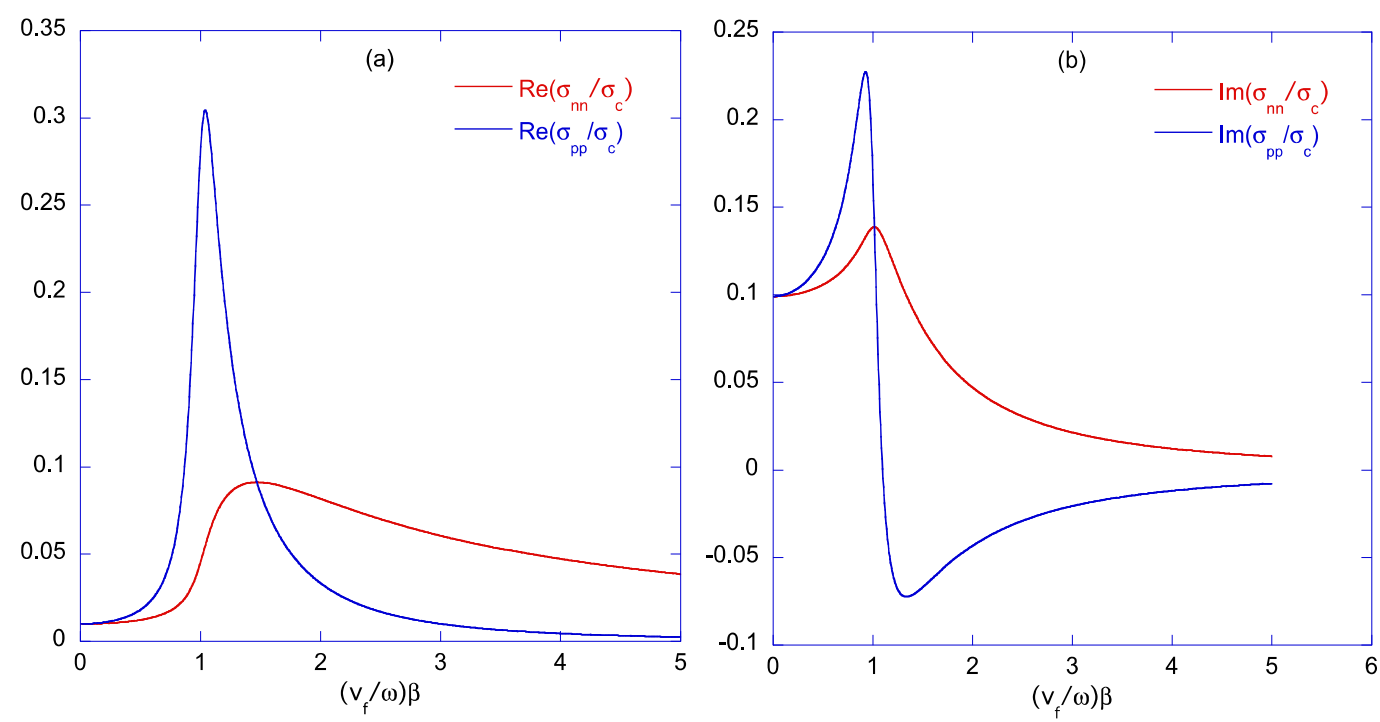

By using Equation (27), it is easy to show that there are off-diagonal terms of the dyad; hence, in general, $\sigma_{x y}(\omega, \boldsymbol{\beta})=\sigma_{y x}(\omega, \boldsymbol{\beta}) \neq 0$. The dyadic nature of the conductivity introduces a spatial dispersion, which may be easily observed from Figures 8 and 9, which show the real and imaginary parts of $\sigma_{|| \mid}(\omega, \boldsymbol{\beta})$ and $\sigma_{\perp \perp}(\omega, \boldsymbol{\beta})$, normalized to $\sigma_{c}$, versus $\left(v_{F} / \omega\right) \beta$ for $v / \omega=0.1$ and $v / \omega=1$, respectively. It is evident that a resonance still arises at $\omega \cong \beta v_{F}$ in both cases.

Figure 9. Real (a) and imaginary (b) parts of $\sigma_{\|||}(\omega, \boldsymbol{\beta})$ and $\sigma_{\perp \perp}(\omega, \boldsymbol{\beta})$, normalized to $\sigma_{c}$, vs. $\left(v_{F} / \omega\right) \beta$ for $v / \omega=1$.
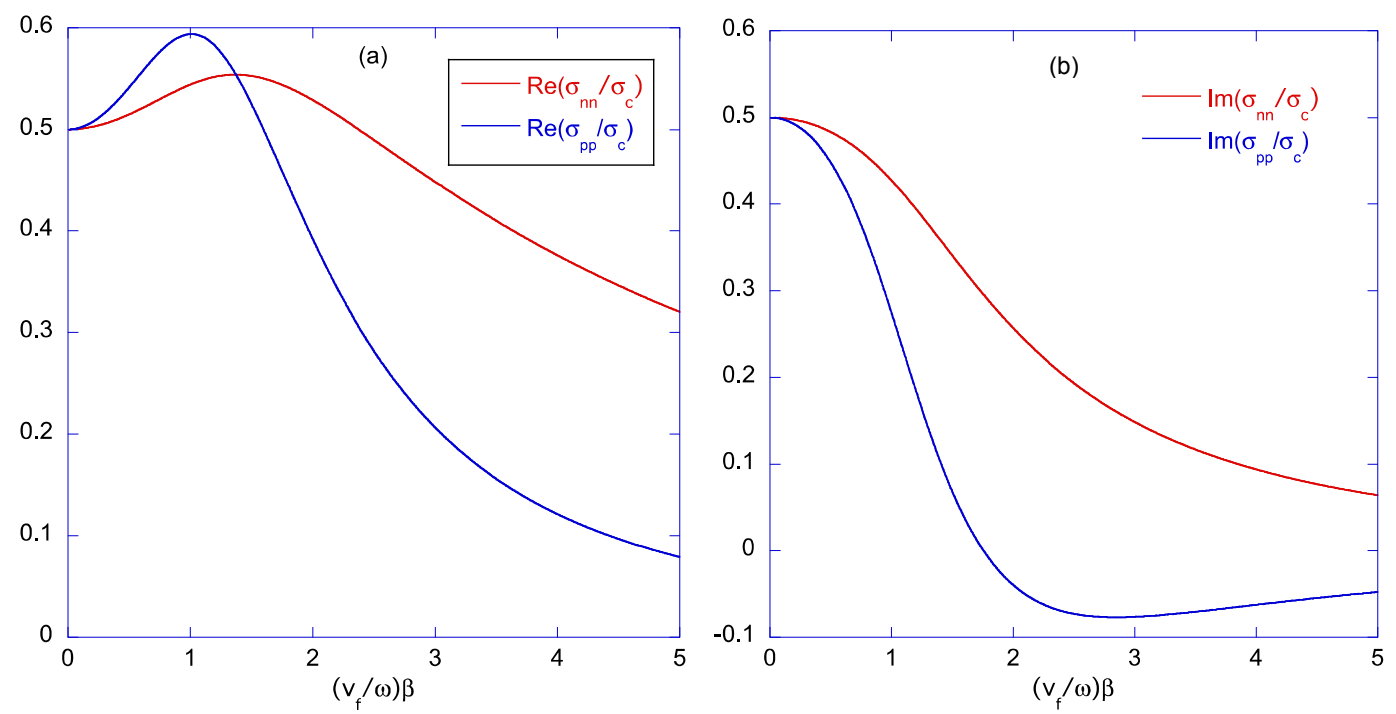
The relation between the dyadic nature of the conductivity and the spatial dispersion is consistent with the result obtained in [25], where the graphene conductivity dyad is derived from the Kubo formula and numerically evaluated with an integral formulation.

\section{Conclusions}

In the operating conditions predicted for future electronic nano-interconnects, the low bias and the low frequency allows the modeling of the electrical transport with a linearized semi-classical electrodynamical model, which does not include interband transitions. This leads to a generalized non-local dispersive Ohm's law, which is regarded as the constitutive equation for the material. In the 2D case (graphene layer), the conductivity is dyadic and shows a spatial dispersion as an effect of the coupling between the components. This coupling is absent in the long-wavelength limit. In the 1D case (graphene nanoribbon), the transverse components of the currents are negligible, and the conductivity reduces to a scalar quantity, modulated by the size and chirality, which strongly affect the number of conducting channels.

\section{Acknowledgments}

This work has been supported by the EU grant \# FP7-247007, under the project CACOMEL, "Nano-CArbon based COmponents and Materials for high frequency ELectronics".

\section{Conflicts of Interest}

The authors declare no conflict of interest.

\section{References}

1. Van Noorden, R. Moving towards a graphene world. Nature 2006, 442, 228-229.

2. Avouris, P.; Chen, Z.; Perebeinos, V. Carbon-based electronics. Nat. Nanotechnol. 2007, 2, 605-615.

3. Li, H.; Xu, C.; Srivastava, N.; Banerjee, K. Carbon nanomaterials for next-generation interconnects and passives: Physics, status, and prospects. IEEE Trans. Electron Devices 2009, $56,1799-1821$.

4. International Technology Roadmap for Semiconductors, Edition 2013. Available online: http://public.itrs.net (accessed on 23 May 2014).

5. Close, G.F.; Yasuda, S.; Paul, B.; Fujita, S.; Wong, H.-S.P. 1 GHz integrated circuit with carbon nanotube interconnects and silicon transistors. Nano Lett. 2009, 8, 706-709.

6. Chen, X.; Akinwande, D.; Lee, K.-J.; Close, G.F.; Yasuda, S.; Paul, B.C.; Fujita, S.; Kong, J.; Wong, H.-S.P. Fully integrated graphene and carbon nanotube interconnects for gigahertz high-speed CMOS electronics. IEEE Trans. Electron Devices 2010, 57, 3137-3143.

7. Shulaker, M.M.; Hills, G.; Patil, N.; Wei, H.; Chen, H.-Y.; Wong, H.-S.P.; Mitra, S. Carbon nanotube computer. Nature 2013, 501, 526-530.

8. Valitova, I.; Amato, M.; Mahvash, F.; Cantele, G.; Maffucci, A.; Santato, C.; Martel, R.; Cicoira, F. Carbon nanotube electrodes in organic transistors. Nanoscale 2013, 5, 4638-4646. 
9. Burke, P.J. Luttinger liquid theory as a model of the gigahertz electrical properties of carbon nanotubes. IEEE Trans. Nanotechnol. 2002, 1, 129-144.

10. Salahuddin, S.; Lundstrom, M.; Datta, S. Transport effects on signal propagation in quantum wires. IEEE Trans. Electron Devices 2005, 52, 1734-1742.

11. Raychowdhury, A.; Roy, K. Modelling of metallic carbon-nanotube interconnects for circuit simulations and a comparison with $\mathrm{Cu}$ interconnects for scaled technologies. IEEE Trans. Comp.-Aided Des. Integr. Circuit Syst. 2006, 25, 58-65.

12. Maffucci, A.; Miano, G.; Villone, F. Performance comparison between metallic carbon nanotube and copper nano-interconnects. IEEE Trans. Adv. Packag. 2008, 31, 692-699.

13. Xu, C.; Li, H.; Banerjee, K. Modeling, analysis, and design of graphene nano-ribbon interconnects. IEEE Trans. Electron Devices 2009, 56, 1567-1578.

14. Naeemi, A.; Meindl, J.D. Compact physics-based circuit models for graphene nanoribbon interconnects. IEEE Trans. Electron Devices 2009, 56, 1822-1833.

15. Cui, J.-P.; Zhao, W.-S.; Yin, W.-Y.; Hu, J. Signal transmission analysis of multilayer graphene nano-ribbon (MLGNR) interconnects. IEEE Trans. Electromagn. Compat. 2012, 54, 126-132.

16. Maffucci, A.; Miano, G.; Villone, F. A transmission line model for metallic carbon nanotube interconnects. Int. J. Circuit Theory Appl. 2008, 36, 31-51.

17. Maffucci, A.; Miano, G.; Villone, F. A new circuit model for carbon nanotube interconnects with diameter-dependent parameters. IEEE Trans. Nanotechnol. 2009, 8, 345-354.

18. Miano, G.; Forestiere, C.; Maffucci, A.; Maksimenko, S.A.; Slepyan, G.Y. Signal propagation in single wall carbon nanotubes of arbitrary chirality. IEEE Trans. Nanotechnol. 2011, 10, 135-149.

19. Forestiere, C.; Maffucci, A.; Maksimenko, S.A.; Miano, G.; Slepyan, G.Y. Transmission Line model for multiwall carbon nanotubes with intershell tunneling. IEEE Trans. Nanotechnol. 2012, $11,554-564$.

20. Maffucci, A.; Miano, G. Transmission line model of graphene nanoribbon interconnects. Nanosci. Nanotechnol. Lett. 2013, 5, 1207-1216.

21. Forestiere, C.; Maffucci, A.; Miano, G. Hydrodynamic model for the signal propagation along carbon nanotubes. J. Nanophotonics 2010, 4, 041695.

22. Zheng, H.; Wang, Z.F.; Luo, T.; Shi, Q.W.; Chen, J. Analytical study of electronic structure in armchair graphene nanoribbons. Phys. Rev. B 2007, 75, 165414.

23. Wakabayashi, K.; Sasaki, K.; Nakanishi, T.; Enoki, T. Electronic states of graphene nanoribbons and analytical solutions. Sci. Technol. Adv. Mater. 2010, 11, 054504.

24. Chiariello, A.G.; Maffucci, A.; Miano, G. Circuit models of carbon-based interconnects for nanopackaging. IEEE Trans. Compon. Packag. Manuf. Technol. 2013, 3, 1926-1937.

25. Araneo, R.; Lovat, G.; Burghignoli, P. Graphene nanostrip lines: Dispersion and attenuation. In Proceedings of 16th IEEE Workshop on Signal and Power Integrity, Sorrento, Italy, 13-16 May 2012; pp. 75-78.

(C) 2014 by the authors; licensee MDPI, Basel, Switzerland. This article is an open access article distributed under the terms and conditions of the Creative Commons Attribution license (http://creativecommons.org/licenses/by/3.0/). 